\title{
Atmospheric turbulence profiling with SLODAR using multiple adaptive optics wavefront sensors
}

\author{
Lianqi Wang, ${ }^{1, \star}$ Matthias Schöck, ${ }^{1}$ and Gary Chanan ${ }^{2}$ \\ ${ }^{1}$ Thirty Meter Telescope Project, Pasadena, California 91125, USA \\ ${ }^{2}$ Department of Physics and Astronomy, University of California, Irvine, Irvine, California 92697, USA \\ *Corresponding author: lianqiw @tmt.org
}

Received 4 September 2007; revised 20 December 2007; accepted 25 January 2008; posted 31 January 2008 (Doc. ID 87084); published 4 April 2008

\begin{abstract}
The slope detection and ranging (SLODAR) method recovers atmospheric turbulence profiles from time averaged spatial cross correlations of wavefront slopes measured by Shack-Hartmann wavefront sensors. The Palomar multiple guide star unit (MGSU) was set up to test tomographic multiple guide star adaptive optics and provided an ideal test bed for SLODAR turbulence altitude profiling. We present the data reduction methods and SLODAR results from MGSU observations made in 2006. Wind profiling is also performed using delayed wavefront cross correlations along with SLODAR analysis. The wind profiling analysis is shown to improve the height resolution of the SLODAR method and in addition gives the wind velocities of the turbulent layers. (C) 2008 Optical Society of America
\end{abstract}

OCIS codes: $\quad 010.1330,010.7350,110.1085$.

\section{Introduction}

Accurate knowledge of the atmospheric turbulence profile is important for the design and operation of multiconjugate adaptive optics (MCAO) and other advanced adaptive optics (AO) systems [1,2]. In addition, wind speed and direction information for the turbulent layers (in this paper we use the term "layers" for local maxima of the turbulence profile) might help to compensate for the frame delay ( 1-2 frames) of wavefront corrections with $\mathrm{AO}$ systems.

Slope detection and ranging (SLODAR) is a turbulence profiling method that utilizes wavefront gradient measurements from Shack-Hartmann wavefront sensors (SHWFSs) [3,4]. It has the potential to work in tandem with $\bar{M}$ CAO systems. In the SLODAR analysis, cross correlations of wavefront measurements from two stars are used to estimate the relative strengths of turbulent layers at different altitudes. The height resolution and range depend on the number of subapertures in the SHWFS and the

0003-6935/08/111880-13\$15.00/0

(C) 2008 Optical Society of America angular separation of the binary stars used. SLODAR was first used to measure turbulence profiles by the Centre for Advanced Instrumentation (CfAI), Durham at the Observatorio del Roque de los Muchachos, La Palma [5]. It has also been implemented on a portable and stand-alone profiler for the European Southern Observatory (ESO) using a $40 \mathrm{~cm}$ telescope. Various other implementations are described in the literature [6,7].

Most of the conventional SLODAR profiling systems utilize a single SHWFS to measure wavefront slopes simultaneously from binary stars. This requires the separation between the binary stars to be in an appropriate range so that the stars are within the field of view of the SHWFS and their images are also well-separated in the subapertures. For SLODAR profiling to work with MCAO systems, it has to be expanded to multiple and independent SHWFSs. The Palomar multiple guide star unit (MGSU) [8] has four SHWFSs and provides an ideal testbed for evaluating the performance of SLODAR with SHWFSs. We carried out our SLODAR analysis using all six possible baselines between the four SHWFSs.

We also did a wind profiling analysis, using timedelayed cross correlations of MGSU data. The peaks 
in the delayed cross-correlation frames are dependent upon the time delay. By tracking the movement of these peaks with increasing time delay, we are able to determine the layer altitudes, strengths, and wind velocities. We also show that the wind profiling method is able to detect more layers than the conventional method.

The MGSU was only operated at Palomar during three brief engineering runs with the main purpose of performing quantitative tests of tomographic turbulence estimation. The data analyzed in this paper are by-products of one of these tests. While they are not optimized for SLODAR measurements, they are the only data currently available. Their analysis therefore requires more care than a cleaner dataset would need. In describing the steps required for a successful turbulence and wind profiling analysis using these multiple wavefront sensor data, we hope to provide guidelines for future experiments and applications as to which aspects of the setup, observation, and analysis need to be taken into account.

The MGSU experiment is described below in Section 2 and the data reduction methods are presented in Section 3. SLODAR profiling and its comparison with multi aperture scintillation sensor (MASS) results are shown in Section 4. Finally, a detailed wind profiling analysis is presented in Section $\underline{5}$.

\section{MGSU Experimental Setup}

The Palomar MGSU [8] was developed to test tomographic turbulence estimation using multiple natural guide stars and SHWFSs on the Palomar 200 in. telescope. The Palomar MGSU features four CCD-based SHWFSs, referred to as MGSUs 1-4. All four SHWFSs have $16 \times 16$ square subaperture channels $(0.31 \mathrm{~m} \times 0.31 \mathrm{~m}$ on the primary mirror $)$ and use identical $64 \times 64$ frame transfer CCD cameras $\left(0.75 "\right.$ square pixels, $\sim 5 e^{-}$readout noise) and controllers. MGSU 1 is binned on-chip to $32 \times$ 32 pixels and acts as the active high-order wavefront sensor to feed the tip-tilt (TT) and high-order deformable mirror (DM) loops of the Palomar AO system in normal operation. As a result, the MGSU 1 camera has only $2 \times 2$ CCD pixels in each subaperture, while MGSUs $2-4$ each have $4 \times 4$ CCD pixels per subaperture. Due to differences in the MGSU camera light paths, each camera has a different orientation when projected onto the sky.

An open loop (to be precise, the DM loop is open and the TT loop is closed) MGSU engineering run was carried out during the nights of 9 and 10 February 2006. Four stars from an asterism in SAO 23181 were observed by the four MGSU cameras. Table 1 gives the names and coordinates of the stars observed during the experiments as well as their magnitudes. Angular distances between the stars are listed in Table 2. There are five successful data acquisitions through the night of 10 February 2006 and the analysis presented here is based on those data. Table 3 gives information about the five datasets. The MGSU experiment was originally designed to test tomographic turbulence estimation; we use the

Table 1. Names and Coordinates (Epoch J2000.0) of the Four Stars Observed

\begin{tabular}{cccr}
\hline Star & Name & Right Ascension & Declination \\
\hline 1 & $\mathrm{BD}+56527$ & 021910.46 & +570750.6 \\
2 & $\mathrm{BD}+56526$ & 021910.26 & +570738.3 \\
3 & $\mathrm{BD}+56529$ & 021913.62 & +570743.4 \\
4 & $\mathrm{BD}+56525$ & 021909.28 & +570806.3 \\
\hline
\end{tabular}

Table 2. Star Angular Separations $(\theta)$ of the Six Baselines and the Corresponding Height Resolution $\delta h$ and Range $H_{\text {max }}{ }^{a}$

\begin{tabular}{|c|c|c|c|}
\hline Baseline & $\theta(\operatorname{arcsec})$ & $\delta h(\mathrm{~km})$ & $H_{\max }(\mathrm{km})$ \\
\hline $1-2$ & 12.4 & $5.2 / \sec \zeta$ & $78.0 / \sec \zeta$ \\
\hline $1-3$ & 26.7 & $2.4 / \sec \zeta$ & $36.2 / \sec \zeta$ \\
\hline $1-4$ & 18.4 & $3.5 / \sec \zeta$ & $52.5 / \sec \zeta$ \\
\hline $2-3$ & 27.8 & $2.3 / \sec \zeta$ & $34.8 / \sec \zeta$ \\
\hline $2-4$ & 29.1 & $2.2 / \sec \zeta$ & $33.2 / \sec \zeta$ \\
\hline $3-4$ & 42.1 & $1.5 / \sec \zeta$ & $23.0 / \sec \zeta$ \\
\hline
\end{tabular}

${ }^{a}$ See Eq. (יㅜ).

Table 3. Five Successful Datasets from the Experiments, All of Which Are From 10 February $2006^{a}$

\begin{tabular}{ccc}
\hline Time $(\mathrm{UT})$ & Frame Rate $(\mathrm{Hz})$ & Airmass \\
\hline $03: 35$ & 256 & 256 \\
$03: 55$ & 80 & 1.27 \\
$05: 35$ & 200 & 1.70 \\
$06: 08$ & 128 \\
$06: 40$ & 1.80 \\
\hline
\end{tabular}

${ }^{a}$ The duration of each dataset is approximately $5 \mathrm{~min}$. 
dataset here to test the SLODAR method using multiple wavefront sensors. The steps required for this analysis are described in Section [3].

\section{MGSU Data Analysis}

Conventional SLODAR experiments utilize a single wavefront sensor to collect light from double stars and measure two wavefronts accordingly (two star images in each subaperture). By comparison, the Palomar MGSU has four wavefront measurements, each pair of which can be used as a SLODAR input to recover atmospheric turbulence profiles. The SLODAR analysis presented in this paper uses different pairs (six baselines in total) of the wavefront measurements to obtain different height resolutions and ranges (due to different star separations). By combining SLODAR results from the six different baselines, we are able to cross-check the results and measure the atmospheric turbulent profile to a better resolution and height range combination than can be achieved with SLODAR using a single baseline.

Figures 1 and 2 summarize the main steps involved in the MGSU data analysis. The raw data from the CCD cameras first have the sky background removed, then the images are rotated-flipped according to the orientation alignment information.

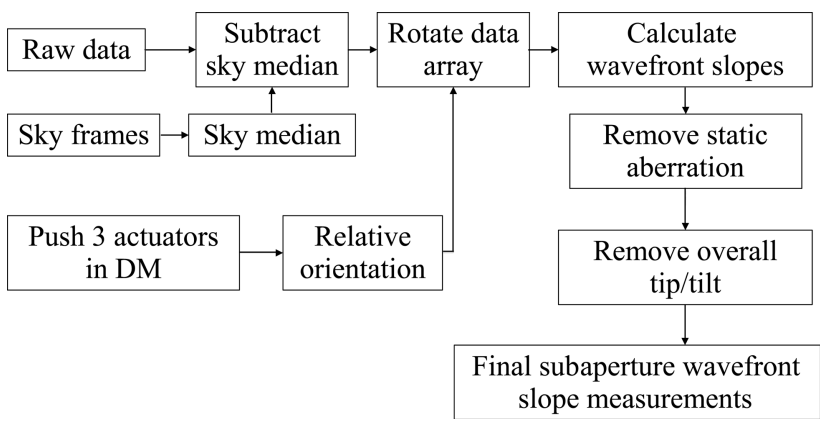

Fig. 1. Flow chart of the wavefront gradient calculation.

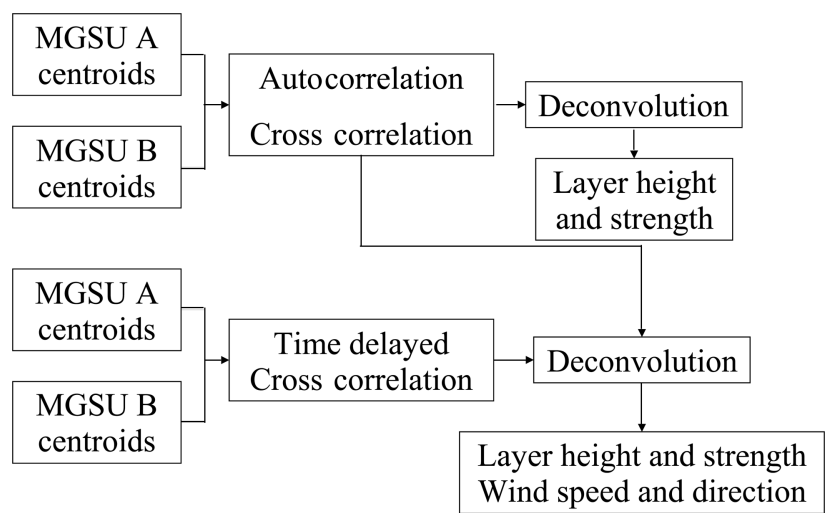

Fig. 2. Flow chart of the SLODAR and wind profiling analysis. MGSUs A and B refer to any pair of MGSU cameras.
Wavefront gradients are calculated and then conditioned by removing the static aberrations and global TT (calculated as the average of the TT of all illuminated subapertures). Atmospheric turbulence profiles are obtained by cross correlating two simultaneous wavefront measurements from any two of the four MGSU cameras. Time-delayed cross correlations are also carried out to estimate the turbulent layer wind speed and direction. The latter step turns out to improve the resolution of the turbulence profile greatly. Details of each step are described in Subsections 3.A and 3.B.

\section{A. Preprocessing}

For the SLODAR analysis as well as for the tomographic turbulence estimation, it is critical to obtain simultaneous wavefront measurements from the MGSU cameras. The instrumental synchronization was carried out during the experiments by sending clock signals from a central source to all cameras simultaneously. The synchronization is also verified during data processing by comparing time stamps of each individual frame. This last step turned out to be necessary as several cases of frame loss were discovered.

The orientations of the MGSU cameras projected onto the sky are different due to differences in the optics in front of each camera. The orientations are verified by applying an asymmetric actuator "poke" pattern on the DM (by pushing up three actuators) and comparing corresponding patterns in all MGSU cameras. The frame orientation alignment is done by flipping the MGSU $1 x$ and $y$ axes and by rotating the MGSU 2 and 4 frames $90^{\circ}$ counterclockwise. The frame orientation alignment is carried out for all data before doing any further analysis.

A series of sky exposures of $\sim 20 \mathrm{~s}$ were taken right before each data acquisition by slewing the telescope several arcseconds away from the stars in order to determine the sky background and CCD dark current. Each data acquisition took approximately $5 \mathrm{~min}$. The median of the sky frames is subtracted from the data frames to compensate for sky background and CCD dark current. As an example, for the MGSU 2 UT 06:08 dataset, the sky background exposure has a median pixel reading of $1665 e^{-}$while the background removed data have pixel readings $\sim 50 e^{-}$near peaks and an average of $13 e^{-}$.

\section{B. Centroids Calculation}

As noted above, the MGSU 1 camera has only $2 \times 2$ CCD pixels in each subaperture because it was originally deployed in the Palomar AO system as the high-speed wavefront sensor. We use the standard center of mass (intensity) centroid method to calculate its wavefront gradients. These are then rescaled according to the transfer function measured in a separate calibration process. This is illustrated in 

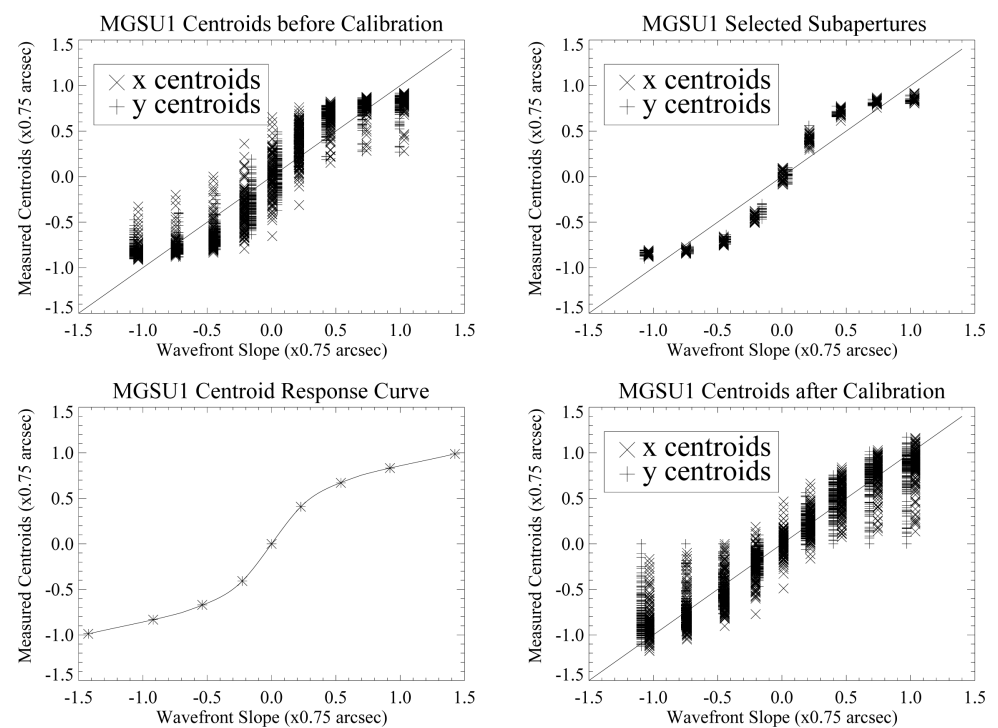

Fig. 3. Top left panel shows the original response of the centroids in each subaperture to the global wavefront tilt set by the TT mirror during the calibration process for MGSU 1. Significant scatter of the measured centroids across the subapertures for a given global wavefront tip-tilt is caused by cross talk between subapertures and optical aberrations. To work around this problem, certain subapertures, which are well-illuminated and have less cross talk or aberration, are selected (top right panel) to construct the response curve of centroids to wavefront gradients (bottom left panel). Centroid results from experimental data are then rescaled based on this response curve. The bottom right panel shows the much improved linearity achieved with the rescaled centroids. The rescaled data show more scatter when the wavefront slope is large because the slope in the response curve is smaller and thus scatter in centroids causes more scatter in measured wavefront slopes.

Fig. 3. The top left panel shows the original response of the centroids in each subaperture to the global wavefront tilt set by the TT mirror. Significant scatter of the measured centroids across the subapertures for a given global wavefront TT is caused by cross talk between subapertures and by optical aberrations. To work around this problem, certain subapertures, which are well-illuminated and have less cross talk or smaller aberrations, are selected (top right panel) to construct the response curve of centroids to wavefront gradients (bottom left panel). Centroid results from the data are then rescaled based on this response curve. The results are shown in the bottom right panel. Although there is still a significant amount of scatter, the averaged centroids are now close to linearly proportional to the actual wavefront slopes.

The other three cameras all have $4 \times 4$ pixels in each subaperture and due to the static aberrations and cross talk between subapertures (no guard cell is present in the MGSU CCDs), the averaged image center is generally not in the center of each nominal subaperture. There are significant amounts of noise present in the data (in large part because the observations were carried out during full-moon nights), which cannot effectively be removed by the subtraction of the sky frame. The above effects cause the standard center of mass centroid calculation to have a very limited range that is not representative of the real wavefront gradients (see Fig. 4) and does not meet our needs. To solve the static aberration problem, we instead divide each CCD frame into subapertures of various sizes according to the intensity distribution in the time averaged CCD frame (see
Fig. 5). The divisions are different for different MGSU cameras and different runs, but stay the same for each camera during consecutive exposures in each run. This figure also shows that the fluxes in subapertures are not uniform (similar to other MGSU cameras). The mean flux is $334 e^{-}$with a standard deviation of $81 e^{-}$. This is partly due to vignetting by the secondary mirror support structure and maybe some other vignetting in the MGSU light path. It should, however, not have a significant effect on the centroid measurements.

We have tried various methods to obtain wavefront gradients in addition to the simple center of mass method, including the iterative boxed center of mass method and the "Find" method combined with the "gcntrd" subroutine in the DAOPHOT package [9]. For the simple center of mass method, due to low signal-to-noise ratio and nonnegligible background residuals, the magnitudes of the wavefront gradients are underestimated, resulting in a severely reduced range of calculated centroids. This can be seen from the histogram of centroids for each subaperture (see the top left panel of Fig. 4). The range of calculated centroids is limited to within \pm 0.5 pixels $( \pm 0.375$ arcsec $)$.

The iterative boxed center of mass is different from the simple center of mass method in that it calculates the center of mass centroid in a small region that has a size similar to that of the point-spread function of the subaperture. It then recenters the region on the measured centroid. The procedure is iterated until it converges. The method works well when the signalto-noise ratio is high, but with the noisy MGSU data, the results are unsatisfactory. The centroid 

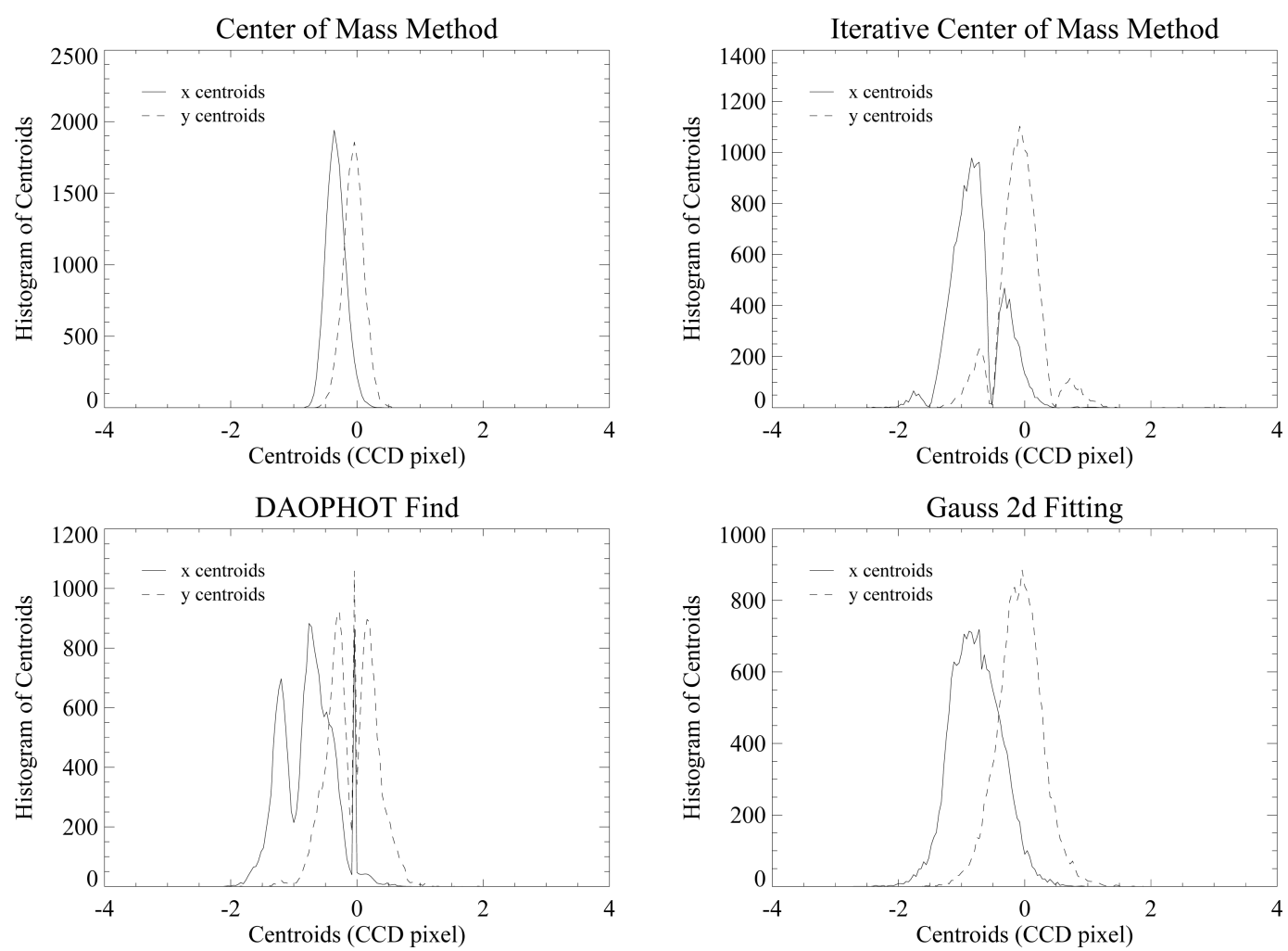

Fig. 4. Comparison of centroid histograms from different centroiding methods (UT 06:40 dataset, MGSU 2, 48000 frame, CCD pixel scale is 0.75 arcsec). Results for a particular subaperture $(4,12)$ are shown. Although the histograms from the center of mass method look Gaussian, the range is severely limited. The histograms from the iterative center of mass method and the DAOPHOT Find method show unphysical departures from a Gaussian shape and are rejected. The histograms from the Gauss two-dimensional fitting have an approximately Gaussian shape and there is no indication of an artificially limited range.

histogram of a typical subaperture is shown in the top right panel of Fig. 4. Although the range of calculated centroids is what is expected, the histogram shows unphysical artifacts (zones of avoidance near half odd integer pixel values) that are probably also caused by noise.

The Find method in the DAOPHOT software package searches for stars in a crowded star frame, and in our case for the star images of each subaperture. The static aberrations cause the star images in each subaperture to move away from the center of the predefined subapertures. Thus the Find method seems to be a good choice to look for these star images and estimate their positions. However, uneven illuminations across the whole frame and poor signal-to-noise ratio cause the method to lose image points frequently and the histogram shows a non-Gaussian distribution, primarily due to the fact that gentrd cannot always determine the precise image center in the presence of noise. As can be seen from the centroid histogram in the bottom left panel of Fig. 4, there are again unphysical artifacts. Even worse is the sharp peak at zero slope, which implies that many of the subapertures are missed in searching for subaperture star images (wavefront slopes are set to zero if no images are found in those subapertures).

All of the above methods suffer from noise and static aberrations and are not satisfactory. We therefore developed a two-dimensional Gaussian fitting

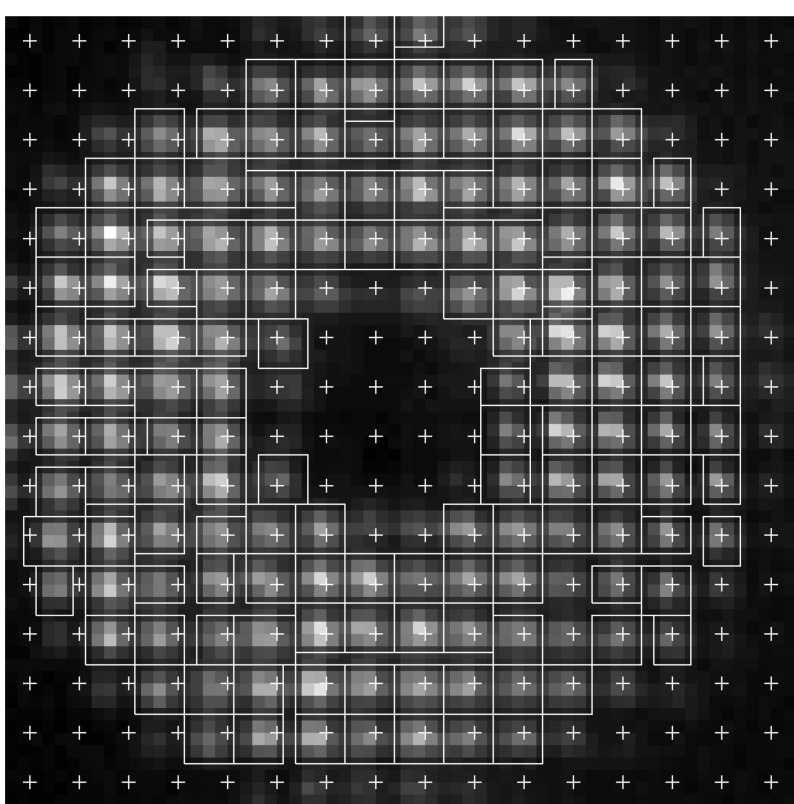

Fig. 5. This example of a time averaged frame shows how subapertures are divided when static aberrations are present (UT 06:40, MGSU 2). The plus signs show the nominal centers of each subaperture; the boxes show how the actual subapertures are divided to calculate wavefront gradients. Due to the static aberrations, nominal and actual subapertures do not agree very well, making this irregular division necessary. Note also that the fluxes in the subapertures are not uniform. The mean flux is $334 e^{-}$with a standard deviation of $81 e^{-}$. 
method that fits all pixels within a subaperture to a two-dimensional Gaussian surface defined by

$$
f(x, y)=a_{0} e^{\left[-\left(x-a_{1}\right)^{2} / a_{2}^{2}\right]-\left[\left(y-a_{4}\right)^{2} / a_{5}^{2}\right]}+a_{3} .
$$

The parameters $a_{1}$ and $a_{4}$ give the wavefront gradients in the $x$ and $y$ directions, respectively. The fitting is carried out following the Levenberg-Marquardt method [10]. An initial center of mass analysis is performed on the subaperture image array to guess the initial values for $a_{1}$ through $a_{5}$, which are then adjusted iteratively to match $f(x, y)$ to the subaperture image array. (In the following, we refer to $a_{1}$ and $a_{4}$ as the Gaussian centroids). The FWHM of the fitted Gaussian surface varies from subaperture to subaperture and from frame to frame. As an example Fig. 6 shows a histogram of the fitted Gaussian surface FWHM for all subapertures of the MGSU 2 UT 06:40 dataset. The distribution shows the expected lognormal shape with a reasonable range of values, thus corroborating that the Gaussian fitting method correctly identifies the images.

The Gaussian centroid histograms we obtained are very nearly Gaussian [11] for most of the subapertures (see the bottom right panel in Fig. 4) and the method proves to be very stable and consistent. The transfer curve calibration also shows a linear response for all three cameras during the calibration process (see Fig. 7).

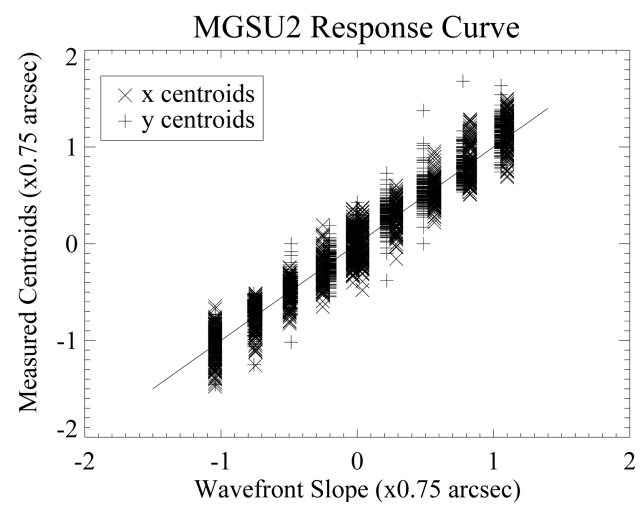

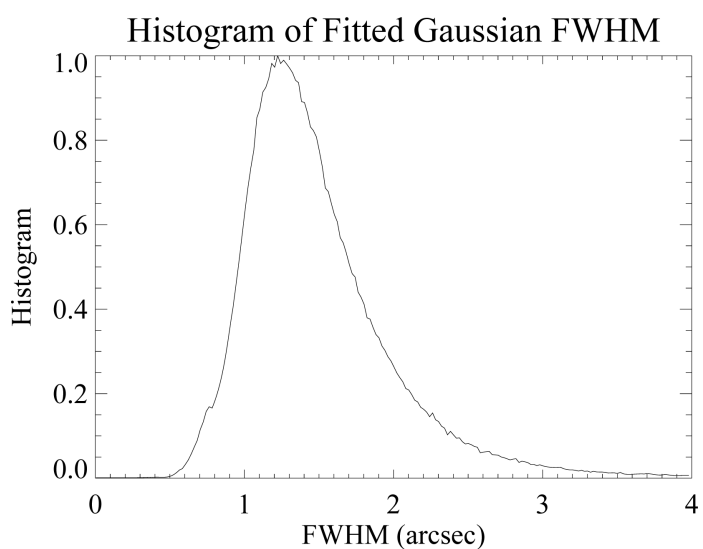

Fig. 6. Histogram of the fitted Gaussian surface FWHM for the MGSU 2 UT 06:40 dataset.

The static aberrations are then removed by subtracting the time averaged centroids in each subaperture. Factors contributing to these static aberrations include the imperfect wavefront correction by the DM and other noncommon path aberrations in the telescope light paths.

A time series of the global wavefront TT for each MGSU camera after the removal of static aberrations is shown in Fig. 8. The common characteristics seen in all four MGSU cameras are largely from

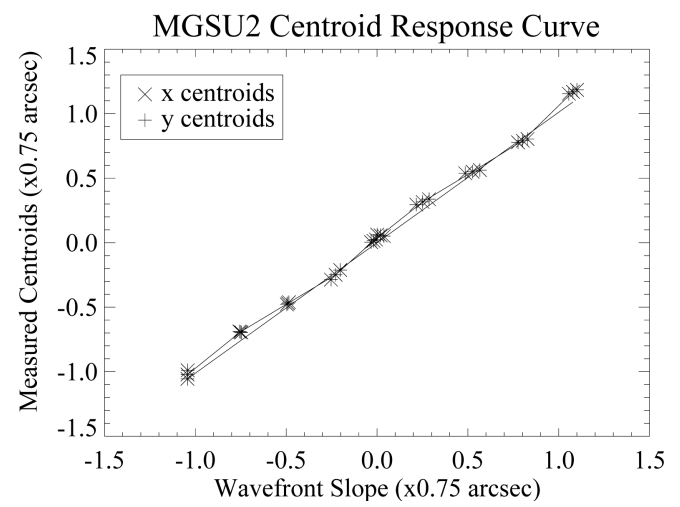

Fig. 7. Left panel: the comparison between the measured centroids and the true wavefront slopes generated by the tip-tilt mirror. Right panel: the comparison between the subaperture averaged centroids and the wavefront slopes. The averaged centroids are close to linearly proportional to the actual wavefront slopes.
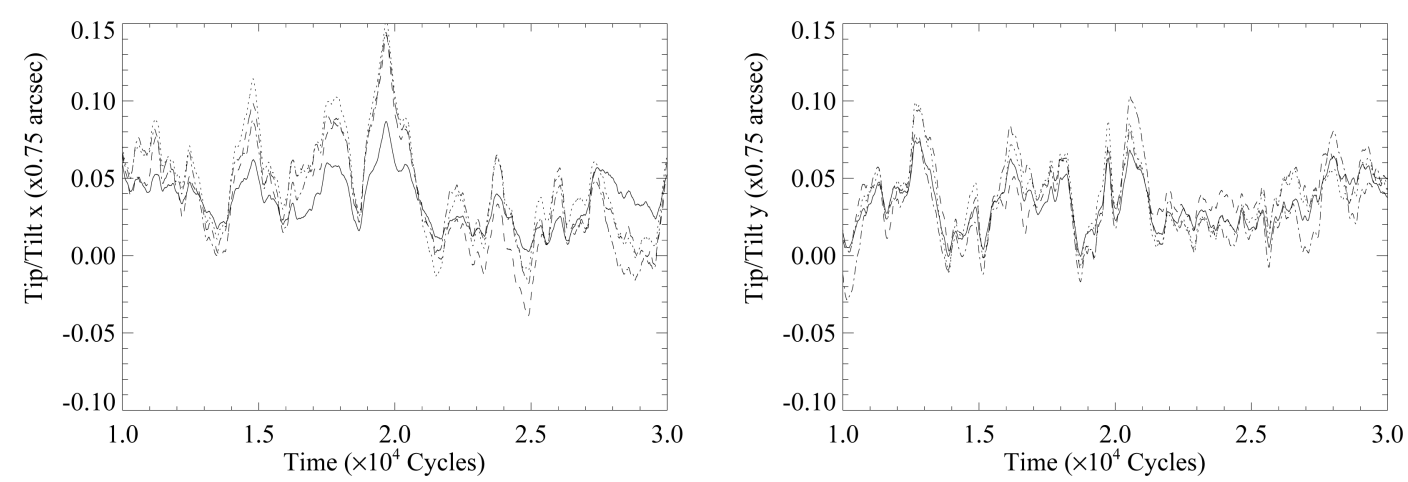

Fig. 8. Left panel: the global tip-tilt in the $x$ direction at UT 03:55 for all four MGSU cameras. Right panel: corresponding $y$ centroids. The curves are smoothed with a boxcar average of 1000 frames. The four TT curves show a similar pattern. This confirms that the synchronization between cameras has been done correctly. 
atmospheric TT. The differences between them are caused by the TT anisoplanatism (difference of TT along different lines of sight). The common features also confirm that the orientation alignment and synchronization are done correctly. As a final step, the global wavefront TT is removed by subtracting the averaged TT value from all illuminated subapertures of each frame.

\section{SLODAR Turbulence Profiling}

A. Introduction

After the centroids are calculated as described above, we take simultaneous wavefront slope measurements from two of the four MGSU cameras and calculate cross correlations using either $x$ or $y$ centroids. Cross correlations using $x$ centroids are referred to as $x$ cross correlations, and similarly for $y$. The cross correlations are calculated using the following formula:

$$
C(\delta i, \delta j)=\frac{\left\langle\sum_{i j} s_{i j}(t) s_{i+\delta i, j+\delta j}^{\prime}(t)\right\rangle}{O(\delta i, \delta j)},
$$

where $s_{i j}(t)$ is the wavefront gradient (in the $x$ or $y$ direction) in subaperture $(i, j)$ at time $t$ for the first camera, and $s_{i+\delta i, j+\delta j}^{\prime}(t)$ is the wavefront gradient in subaperture $(i+\delta i, j+\delta j)$ for the second camera, $\sum_{i j}$ denotes summation over all illuminated subapertures and \langle\rangle means averaging over the time series (all the frames in each dataset); $O(\delta i, \delta j)$ is the number of overlapping illuminated subapertures for offset $(\delta i, \delta j)$, which is largest when $\delta i=0$ and $\delta j=0$, and decreases with increasing $\delta i$ or $\delta j$.

We also carry out autocorrelations for each of the two cameras using the following formula:

$$
A(\delta i, \delta j)=\frac{\left\langle\sum_{i j} s_{i j}(t) s_{i+\delta i, j+\delta j}(t)\right\rangle}{O(\delta i, \delta j)} .
$$

The autocorrelation is an estimate of the "impulse response" of the system to atmospheric turbulence.
In other words, the autocorrelation is determined by the internal properties (such as the structure function) of the turbulence.

The cross correlation is then deconvolved by the autocorrelation [3,4]. We utilize two-dimensional deconvolution methods because the baseline of each pair of cameras is not in general oriented along either the $x$ or $y$ direction. Fast Fourier transform (FFT) deconvolution ( $\left.\mathrm{FT}^{-1}[\mathrm{FT}[C] / \mathrm{FT}[A]]\right)$ is used and proves to be robust if enough frames are averaged. Atmospheric profiles are obtained by a one-dimensional cut of the two-dimensional deconvolution array through its center and in the direction of the alignment of the two stars (see Fig. 9). The layer altitudes $H$ are then converted from the subaperture offset counts $\Delta$ (integer numbers) using

$$
\delta h=\frac{w}{\theta \times \sec \zeta}, \quad H=\Delta \delta h,
$$

where $w$ is the subaperture size, $\theta$ is the angular separation of the two stars, $\zeta$ is the zenith angle of the stars, and $\delta h$ is the altitude resolution. The height resolution and range for the six baselines are shown in Table 2. It can be seen that baselines 1-2 and 1-4, which correspond to the smallest star separation, give poor height resolution but have a higher maximum altitude range.

\section{B. SLODAR Results}

The SLODAR analysis uses the six different baselines between the four MGSU cameras. Each baseline provides the profile of atmospheric turbulence independently, with different resolution and height range because the star separations are different for different baselines. The results from each baseline are shown in Fig. 10 for the UT 06:40 dataset. Turbulence strength $C_{n}^{2} \delta h$ is shown as a function of the altitude $H$. The six curves (in the upper panels) show turbulence profiling results from the SLODAR
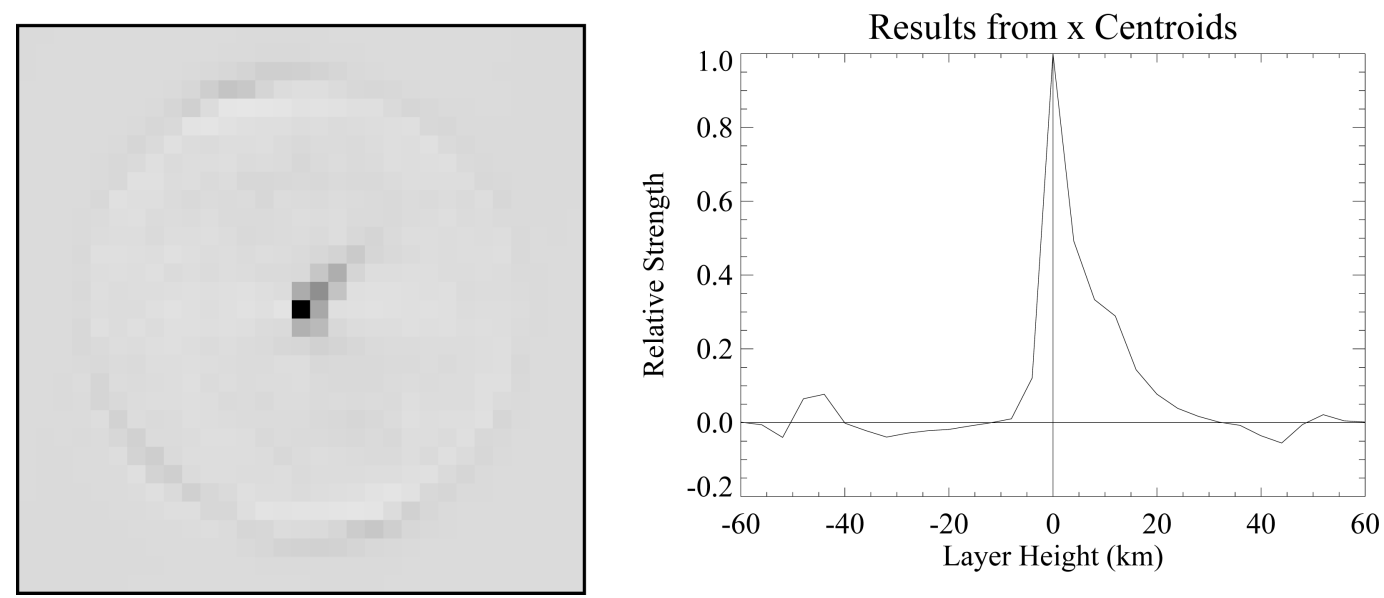

Fig. 9. Left panel shows the two-dimensional deconvolution frame of the cross correlation between MGSUs 1 and 2 for UT03:55, 10 February 2006. Darker areas mean greater turbulence strength. The $x$ and $y$ axes are the directions of the rows and columns of the WFS. The right panel shows a one-dimensional cut of the left panel through the center and along the tail, whose direction is coincident with the direction of the two corresponding guide stars. The right-hand side (height $>0$ ) of the curve shows the turbulence strength profile of the atmosphere. 

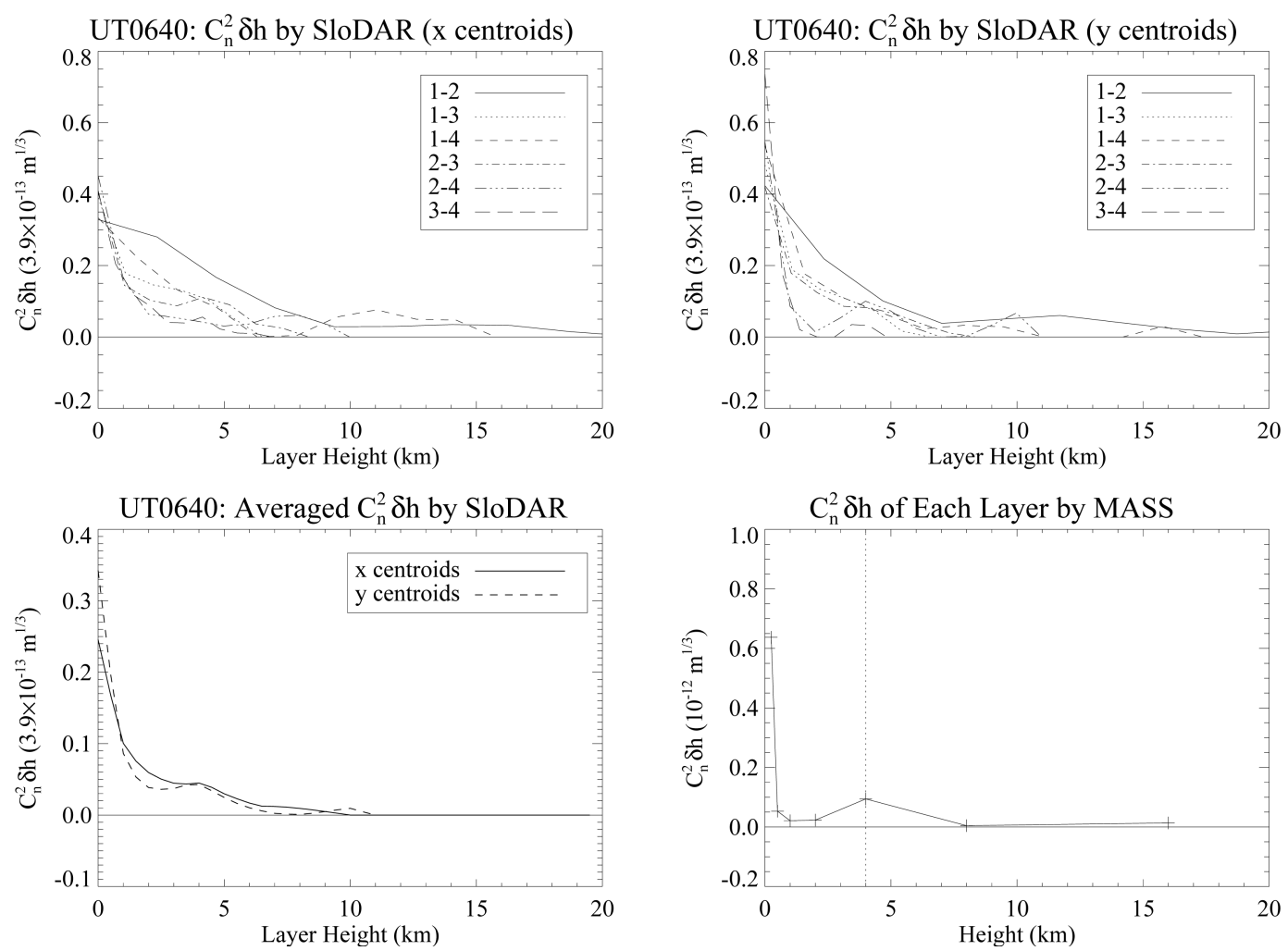

Fig. 10. Top panels: SLODAR results from the UT 06:40 dataset. The six curves in the top plots show turbulence profile results from SLODAR analysis for the six baselines, as indicated in the key in the upper right of each plot. Bottom left panel: the averaged SLODAR results from four different baselines (excluding the short baselines 1-2 and 1-4) with $\delta h=0.5 \mathrm{~km}$. In the averaged data we can clearly see the ground layer and a layer at $4 \mathrm{~km}$. The bottom right panel shows results for the same time (averaged over $\sim 10 \mathrm{~min}$ ) from the Palomar MASs instrument.

analysis for the six baselines. Different baselines have different $\delta h$ [as in Eq. (4)] and can therefore not be compared directly (see below). For each curve the strong oscillations at large heights (mainly $>10 \mathrm{~km}$ ) are due to noise (very few overlapping subapertures at such heights) and should be disregarded (note that negative values in the $C_{n}^{2} \delta h$ curves are nonphysical and are therefore set to zero). As a result, the maximum height that SLODAR profiling can reach in practice is somewhat lower than the theoretical values given in Table 2 .

The apparent differences between the curves for the different baselines are due to the different height resolutions, $\delta h$, and the fact that the plotted quantity is $C_{n}^{2} \delta h$. (Plotting $C_{n}^{2}$ is not practical for the individual baselines as it is not known how the turbulence is distributed within each resolution element.) Baseline 1-2 is the shortest (see Table 2), which leads to a large height range but poor height resolution. Thus, as each data point represents the sum of turbulence over the height range $\delta h$, individual layers (i.e., local maxima of the turbulence profile) cannot be resolved by this pair of wavefront sensors. Baselines 1-4 and 1-3 are the next shortest and show improved resolution but still not enough to resolve the $4 \mathrm{~km}$ layer. However, the short baselines have their own advantages when it comes to wind profiling using delayed cross correlations, as will be shown in Section 5 .
The averaged turbulence profiles from the baselines other than 1-2 and 1-4 are shown in the bottom left panel of Fig. 10. To create these curves, individual profiles were rebinned onto a regular grid with a resolution of $\delta h=0.5 \mathrm{~km}$ and were then averaged. The results obtained from both $x$ and $y$ centroids are shown. The differences between the two curves give an indication of the errors of the analysis, as the $x$ and $y$ centroids can be considered independent datasets. An even better agreement is obtained for the UT 03:55 dataset shown in Fig. 11.

Simultaneous MASS [12] results are also shown for comparison (bottom right panel in Fig. 10; no simultaneous MASS data are available for the other datasets). It needs to be noted that the plotted MASS $C_{n}^{2} \delta h$ has different $\delta h$ for different altitudes, with $\delta h \approx 0.75 \mathrm{~h}$, and that the lowest point is the "ground layer" strength calculated from the difference between the MASS seeing and the seeing of a colocated differential image motion monitor (DIMM). The MASS/DIMM instrument is set up on top of a building more than $100 \mathrm{~m}$ away from the 200 in telescope and at a somewhat lower height. During the experiments, the MASS independently observed different stars than did the MGSU experiments. Despite all these differences, the results from SLODAR and MASS are still comparable. The $4 \mathrm{~km}$ and the ground layer showed up in both SLODAR and MASS results. The relative strength of the $4 \mathrm{~km}$ layer and the ground 

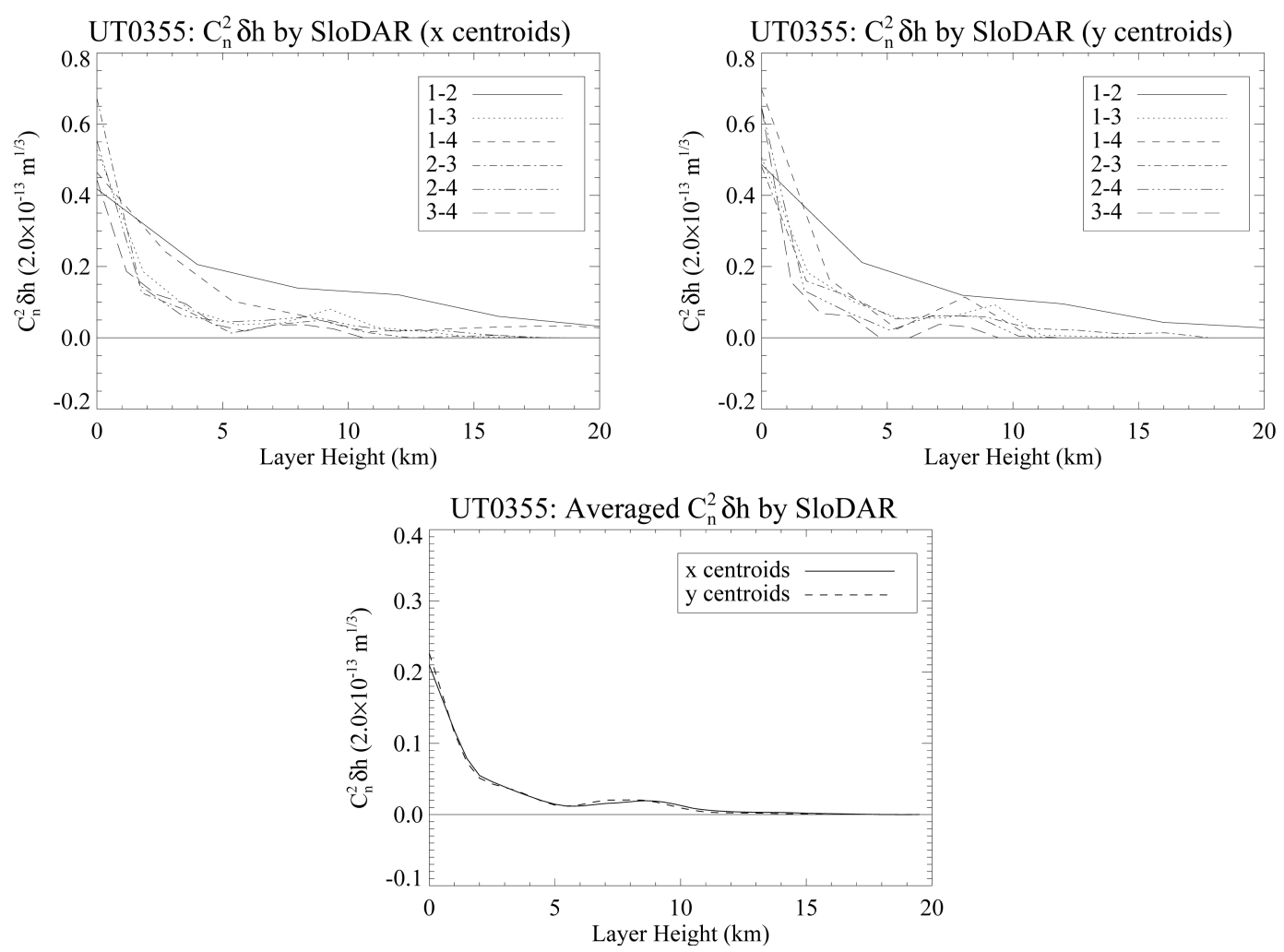

Fig. 11. SLODAR results for the UT 03:55 dataset, as in Fig. 10 but without MASS results because the MASS was not in operation at the same time. In the averaged data we can clearly see the ground layer and a layer around $8 \mathrm{~km}$.

layers is also similar from both results. The absolute strength, in particular of the lowest layer, cannot be compared due to the different locations and different height resolutions of the instruments.

SLODAR results for another dataset (UT 03:55) are shown in Fig. 11. This is similar to Fig. 10 except that the MASS results are from a different time (UT 03:55, 9 February 2006) because the MASS was not in operation at the same time.

\section{Wind Profiling}

\section{A. Introduction}

Wind profiling is carried out on the same data as the SLODAR analysis by performing time-delayed cross correlations between the wavefront measurements of two MGSU cameras using the formula

$$
C(\delta i, \delta j, \delta t)=\frac{\left\langle\sum_{i j} s_{i j}(t) s_{i+\delta i, j+\delta j}^{\prime}(t+\delta t)\right\rangle}{O(\delta i, \delta j)},
$$

where $s_{i j}(t)$ is the $x$ or $y$ component of the wavefront gradient in subaperture $(i, j)$ at time $t$ for the first MGSU camera, $s_{i+\delta i, j+\delta j}^{\prime}(t+\delta t)$ is the wavefront gradient in subaperture $(i+\delta i, j+\delta j)$ at time $(t+\delta t)$ (in our analysis $\delta t$ ranges from $27.3 \mathrm{~ms}$ to $\sim 1 \mathrm{~s}$ and is a multiple of the acquisition time of the corresponding MGSU frame) for the second camera, $\sum_{i j}$ denotes summation over all overlapping illuminated subapertures, \langle\rangle means averaging over the time series, and $O(\delta i, \delta j)$ is the number of overlapping illuminated subapertures for offset $(\delta i, \delta j)$.

The time-delayed cross correlations are then deconvolved by the simultaneous autocorrelations, which are calculated using Eq. (3). When $\delta t$ in Eq. (5) is zero, the time-delayed cross correlation is no different than the simultaneous cross correlation, with the peaks corresponding to different turbulent layers aligned in the direction of the star separation in the deconvolved graph (see Section 2). When $\delta t$ has a positive value, the same peaks show up in the deconvolution graph of the time-delayed cross correlation, but the positions of these peaks move with increasing $\delta t$, depending on the speed of each turbulent layer under the influence of the wind. (The peaks actually move in the opposite direction to the wind.) This time-delayed cross correlation method is mostly sensitive to horizontal movement of the layers and is almost independent of vertical movements. By tracking the movement of the peaks with increasing $\delta t$ we can estimate the wind speed and direction (horizontal component only). Because layers at different altitudes usually have different wind speeds or directions, the peaks corresponding to different layers move with different velocities in the deconvolution graph of the time-delayed cross correlations. In this way, we can identify layers that are too close in altitude to be resolved by the conventional SLODAR methods as long as they do not also have the same wind velocities. We can also identify weak layers that are hard to identify (or verify) by the conventional 
SLODAR analysis due to noise in the deconvolution graph-curve. All of the peaks in the time-delayed deconvolution graph that move with increasing $\delta t$ correspond to turbulent layers with their altitudes, relative strengths (determined from the strength of the peaks belonging to each layer), and wind velocities determined. There usually also exists a peak in the center of the deconvolution graph that does not move with increasing $\delta t$. This peak is caused by the so-called dome-seeing (turbulence within the telescope dome). The altitude resolution of the wind profiling is only restricted by how well one can track the peaks in the deconvolution graph, and can be very high even with a limited number of subapertures. The wind profiling can then greatly improve the height resolution from the conventional SLODAR analysis.

The wind profiling method described above is based on the frozen flow hypothesis, which states that on short time scales the turbulence pattern of each layer is presumed to be fixed (frozen) and to move with a constant speed $[13,14]$. The existence of a frozen flow is important in our analysis because it guarantees that for short time differences the wavefronts $s_{i j}(t)$ and $s_{i j}(t+\delta t)$ are still well correlated. This means that for the increasing time difference $\delta t$ in Eq. (5), the peaks move with significant power remaining in them until $\delta t$ is so large that the frozen flow hypothesis is no longer valid. By estimating how long the peaks persist, it is also possible to estimate the time scale over which the frozen flow assumption is valid.

\section{B. Wind Profiling Results}

Figure 12 shows a selection of the time-delayed cross-correlation frames between MGSUs 1 and 2 with the positions of the peaks marked by plus signs (from the UT 03:55 dataset). The positions of the peaks are determined by fitting the peaks locally to two-dimensional Gaussian surfaces. We can see the peaks corresponding to different layers persist in most of the frames. This implies that a significant part of flow is frozen when the time delay is $\leq 500 \mathrm{~ms}$. The motion of the peaks with increasing $\delta t$ is caused by the motion of the corresponding layers due to horizontal wind. To demonstrate this more clearly, we plot the positions of the peaks overlapped over the nondelayed cross-correlation frame in Fig. 13. We can now clearly see the movement of the peaks with increasing time delay.

We also see that the peaks from the same layer are all approximately aligned in a straight line, which corresponds to the direction of the wind in that layer. The intersection of these lines with the dashed line (the star separation direction) gives the initial position of the layers (marked by diamonds), which are

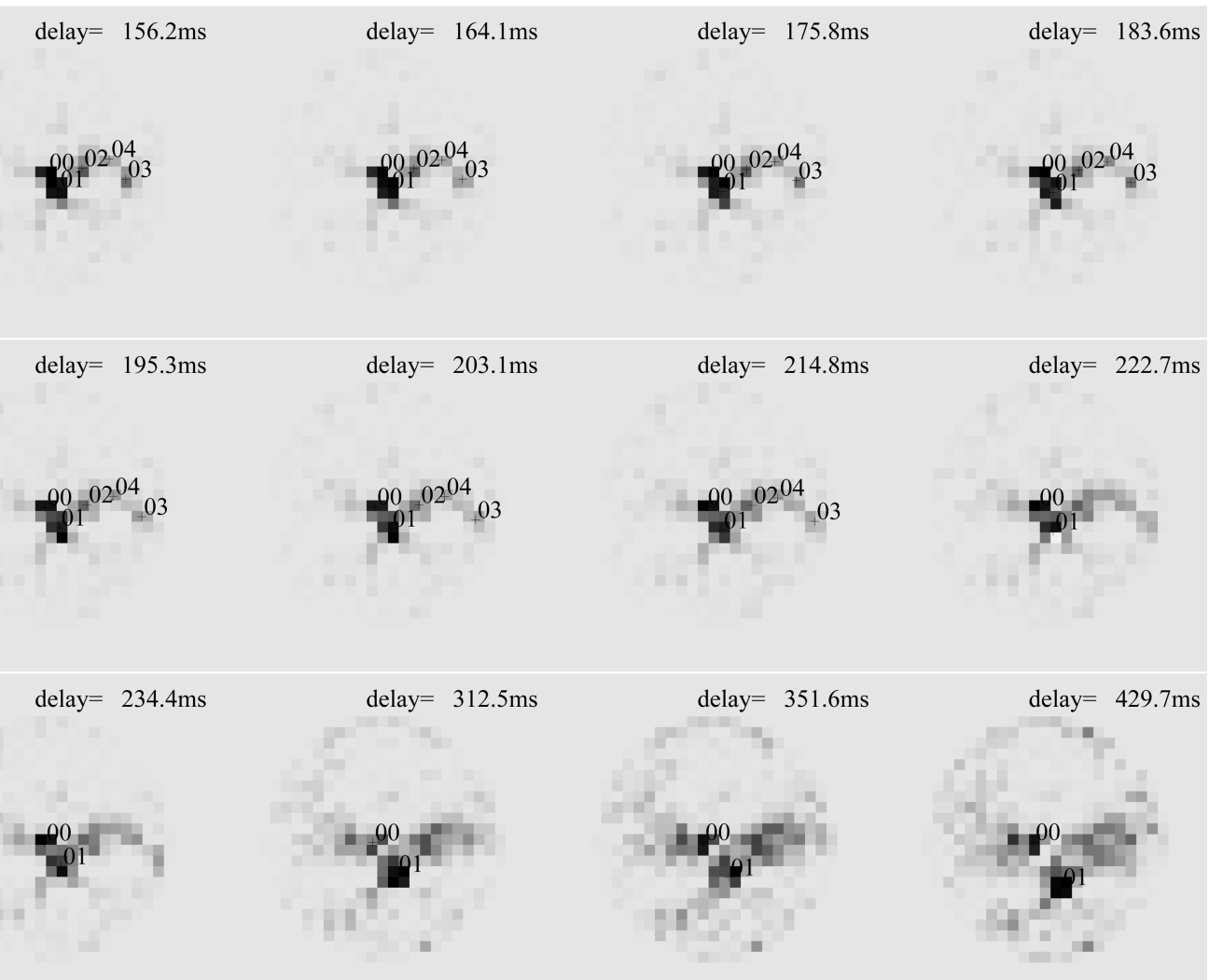

Fig. 12. These figures show a series of time-delayed cross correlations between MGSUs 1 and 2 (from the UT 03:55 dataset). Darker areas mean greater turbulence strength. The peaks corresponding to different layers are marked by plus signs and the layers are numbered 0 through 4 . The positions of the peaks are determined by fitting the peaks locally to two-dimensional Gaussian surfaces. In the last five frames exact positions for peaks 3 and 4 are hard to identify due to spreading and are not shown here. 


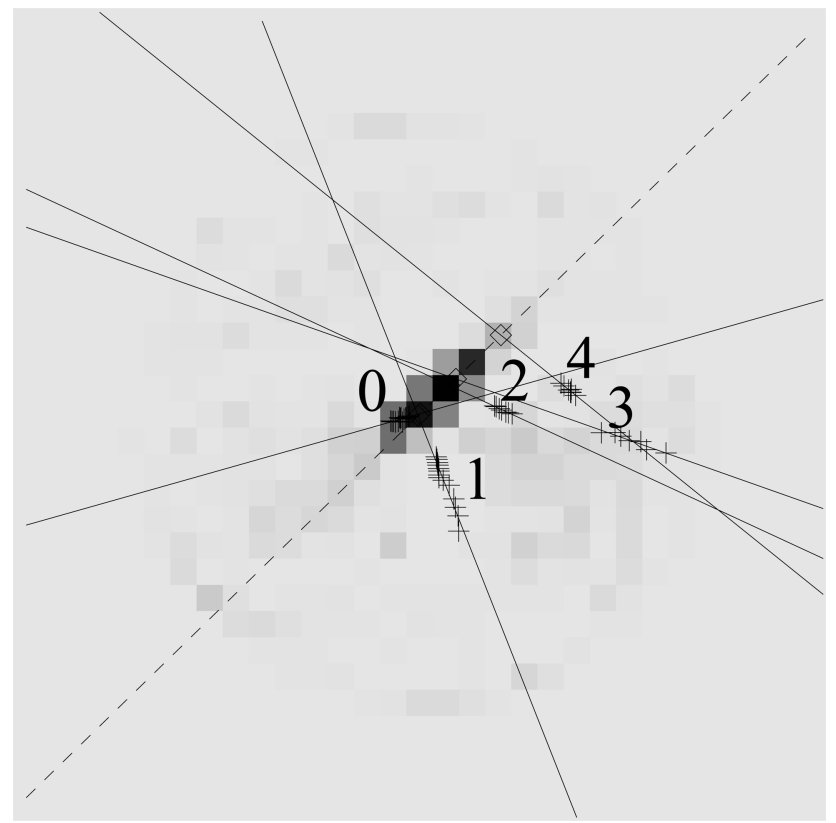

Fig. 13. Positions of the peaks found in Fig. 12 are shown as plus signs. Peaks from the same layer are aligned in a straight line, corresponding to the direction of the wind in these layers (the opposite direction in reality). The intersections of these lines with the dashed line (the star separation direction) give the initial position of the layers (marked by open diamonds), which can be used to calculate the layer heights. The wind speed is calculated by dividing the distance between the plus signs and the respective diamonds by the corresponding delay time $(\sim 150400 \mathrm{~ms})$.

used to calculate their heights. The wind speed is calculated by dividing the distance between the plus signs and the respective diamonds by the corresponding delay time. The wind speed and direction results, as well as the height and strength of these layers, are shown in Table 4 . The tabulated uncertainties reflect the numerical errors from fitting the peaks of each layer to straight lines (as described in Subsection 5.C). The turbulent layer heights and strength results obtained from the wind profiling analysis are plotted against the SLODAR results of the same dataset (as shown in Fig. 11) in Fig. 14. The $8 \mathrm{~km}$ layer seen in the SLODAR results is now identified as two distinct layers with similar altitudes but very different wind speeds. Two distinct layers near the ground (the lower of the two might be a shear layer across the dome opening) and a dome-seeing contribution are also identified with corresponding wind speed and direction, which are not separated by the SLODAR analysis due to limited resolution. An additional layer at $17 \mathrm{~km}$ is also

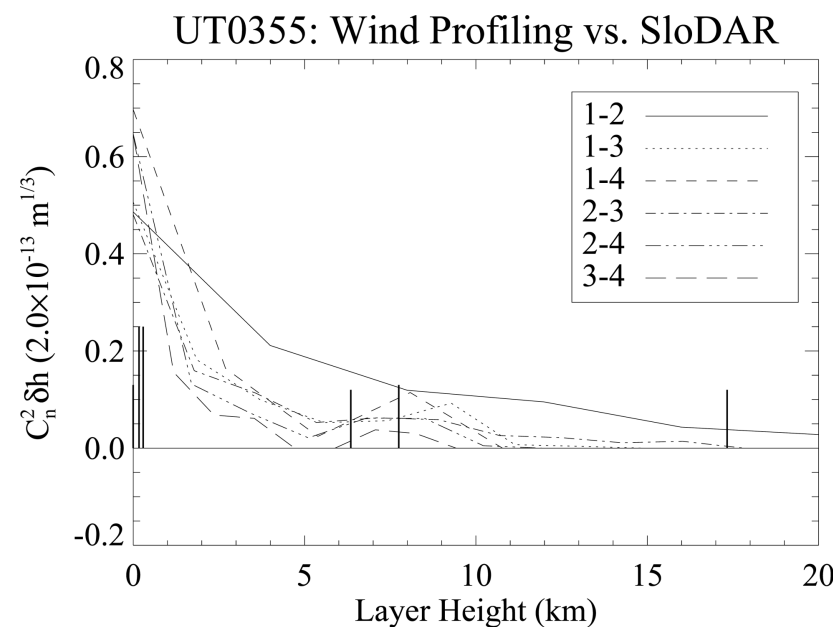

Fig. 14. Wind profiling results are plotted together with the SLODAR results determined for the same data set (see Fig. 11). The six curves as shown in the legend are SLODAR results from the six baselines. The wind profiling results are shown as vertical bars with the relative strength indicated by the bar length. The two layers around $7 \mathrm{~km}$ as seen in the wind profiling analysis, as well as the three ground layers, are not resolved by SLODAR.

Table 4. Five Layers 0-4 Identified by the Wind Profiling Method for the UT 03:55 Dataset, with Their Heights, Relative Strengths, Wind Speeds, and Directions All Determined ${ }^{a}$

\begin{tabular}{lcrrr}
\hline Layer & Strength & Height $(\mathrm{km})$ & Speed $(\mathrm{m} / \mathrm{s})$ & Direction $($ degrees $)$ \\
\hline Dome & $0.13 \pm 0.00$ & $0.00 \pm 0.00$ & $0.00 \pm 0.00$ & $0.0 \pm 0.0$ \\
0 & $0.25 \pm 0.12$ & $0.17 \pm 0.43$ & $0.99 \pm 0.10$ & $215.5 \pm 3.6$ \\
1 & $0.25 \pm 0.12$ & $0.29 \pm 0.35$ & $3.43 \pm 0.07$ & $119.7 \pm 1.6$ \\
2 & $0.12 \pm 0.03$ & $6.35 \pm 0.25$ & $3.78 \pm 0.04$ & $76.2 \pm 1.5$ \\
3 & $0.12 \pm 0.03$ & $7.75 \pm 1.36$ & $12.00 \pm 0.20$ & $70.7 \pm 2.4$ \\
4 & $0.13 \pm 0.03$ & $17.33 \pm 0.87$ & $5.65 \pm 0.27$ & $90.1 \pm 3.7$ \\
\hline
\end{tabular}

${ }^{a}$ The relative strengths are constrained to add up to 1 . Compared to conventional SLODAR analysis, the wind profiling not only can obtain wind speed and direction, but can also greatly improve the height resolution of the layers. 
identified. This layer cannot be identified in the SLODAR analysis because of increased noise in the deconvolution graph at large subaperture offsets. The knowledge of high altitude layers from wind profiling results should help to increase the field angle that an MCAO system can achieve for a given Strehl ratio, since it is mostly limited by the high layer turbulence. The wind speed and direction information can also be applied to wavefront prediction.

\section{Error Analysis}

In Subsection 5.B we referred to the fact that the peaks in Fig. 13 corresponding to a certain layer are fitted to a straight line. This section describes how the fitting and associated error analysis is carried out.

Let the Cartesian spatial coordinates of the peaks in the deconvolution frame be $\left(x_{i}, y_{i}\right)$ for a given layer and let $n$ be the number of peaks. These peaks are fitted to a straight line $y=a^{\prime}+b(x-\bar{x})$. We can obtain $a^{\prime}, b$, and their uncertainties from standard chisquare fitting [10]. Then we can proceed to calculate the layer parameters. Suppose the line of the star separation is $y=a_{0}+b_{0} x$, where $a_{0}$ and $b_{0}$ are defined by the star coordinates and the errors are negligible. Its intersection with the peak movement line $y=$ $a^{\prime}+b(x-\bar{x})$ gives the altitude of the turbulent layer because it describes the position of the peak in the simultaneous cross correlation. Let us call $\left(x_{0}, y_{0}\right)$ the intersection point. We have

$$
y_{0}=a_{0}+b_{0} x_{0}, \quad y_{0}=a^{\prime}+b\left(x_{0}-\bar{x}\right),
$$

and the layer altitude is

$$
H=r \delta h, \quad r=\sqrt{\left(x_{0}-15\right)^{2}+\left(y_{0}-15\right)^{2}},
$$

where $(15,15)$ is the center of the deconvolution frame and $\delta h$ is the altitude corresponding to a one-sub-aperture offset, as defined in Eq. (ㅁ). It is then straightforward to calculate the uncertainty in $H$ from $\delta a^{\prime}$ and $\delta b$. We have

$$
\begin{aligned}
& \frac{\partial H}{\partial a^{\prime}}=\delta h\left(\frac{x_{0}-15}{r}+b_{0} \frac{y_{0}-15}{r}\right) \frac{1}{b_{0}-b}, \\
& \frac{\partial H}{\partial b}=\delta h\left(\frac{x_{0}-15}{r}+b_{0} \frac{y_{0}-15}{r}\right) \frac{x_{0}-\bar{x}}{b_{0}-b} .
\end{aligned}
$$

Keep in mind that $\delta a^{\prime}$ and $\delta b$ have zero covariance. The uncertainty in $H$ is therefore

$$
\begin{aligned}
(\delta H)^{2}= & \left(\frac{\partial H}{\partial a^{\prime}}\right)^{2}\left(\delta a^{\prime}\right)^{2}+\left(\frac{\partial H}{\partial b}\right)^{2}(\delta b)^{2} \\
= & (\delta h)^{2}\left(\frac{x_{0}-15}{r}+b_{0} \frac{y_{0}-15}{r}\right)^{2} \\
& \times\left[\left(\frac{1}{b_{0}-b}\right)^{2} \frac{\sigma^{2}}{n}+\left(\frac{x_{0}-\bar{x}}{b_{0}-b}\right)^{2} \frac{\sigma^{2}}{\sum_{i}\left(x_{i}-\bar{x}\right)^{2}}\right] .
\end{aligned}
$$

The wind direction $\theta$ in degrees is obtained from the parameter $b$ via

$$
\theta=\frac{180}{\pi} a \times \tan b,
$$

and uncertainty in $\theta$ is

$$
\delta \theta=\frac{180}{\pi} \frac{\delta b}{1+b^{2}} .
$$

The wind speed for each layer is obtained from the speed of the peaks in the deconvolution frame. Let $t_{i}$ be the time delay for peak $\left(x_{i}, y_{i}\right)$ and let $d$ be the size of the subaperture when measured at the telescope pupil. The wind speed $v$ will be

$$
v=\left\langle v_{i}\right\rangle=\left\langle\frac{d \sqrt{\left(x_{i}-x_{0}\right)^{2}+\left(y_{i}-y_{0}\right)^{2}}}{t_{i}}\right\rangle,
$$

where $v_{i}$ is the wind speed calculated from peak $\left(x_{i}, y_{i}\right)$ and \langle\rangle denotes averaging over all the peaks for a certain layer. The uncertainty for $v$ is the standard deviation of the $v_{i}$,

$$
\delta v=\sqrt{\frac{\sum_{i=1}^{N}\left(v_{i}-v\right)^{2}}{N-1}},
$$

where $N$ is the number of the available peaks for the layer. The uncertainty for the layer strength is the standard deviation of the strengths determined from all peaks (at different $\delta t$ ) of each layer.

Note that our error analysis does not include the errors in obtaining the positions of the peaks and therefore may be slightly underestimated, but the difference should be small because the positions of the peaks are determined by two-dimensional Gaussian fitting with relatively good accuracy.

\section{Conclusions}

We have demonstrated turbulence profiling analysis with MGSU data using the SLODAR method. The Gaussian centroid determination method works well with the noisy MGSU data and shows good Gaussian distributions and range of centroids. Having six baselines from four guide stars is an advantage over conventional single baseline SLODAR and improves the altitude range and resolution and the overall accuracy.

Wind profiling using time-delayed cross correlations is also performed on the MGSU data. This method tracks the movement of the peaks that correspond to different turbulent layers in the timedelayed cross-correlation frames with increasing time delay between two SHWFS measurements. More turbulent layers are discovered with this method than with SLODAR, and their heights, relative strengths, and velocities are all determined with good accuracy. The height resolution of the wind profiling method is not limited by the number of 
subapertures but is only determined by the accuracy of the determination of the peak positions. An important potential application of such improved height and strength information would be to help improve the three-dimensional turbulence reconstruction in MCAO systems. The wind speed and direction information should be useful in predictive AO corrections.

This work was supported by the Thirty Meter Telescope (TMT) project. The MGSU data were provided by V. Velur (Caltech) and R. C. Flicker (Keck, TMT), and the Palomar AO team. The MAss data were provided by the TMT site-selection team.

We also gratefully acknowledge the support of the TMT partner institutions. They are the Association of Canadian Universities for Research in Astronomy (ACURA), the California Institute of Technology, and the University of California. This work was supported as well by the Gordon and Betty Moore Foundation, the Canada Foundation for Innovation, the Ontario Ministry of Research and Innovation, the National Research Council of Canada, the Natural Sciences and Engineering Research Council of Canada, the British Columbia Knowledge Development Fund, the Association of Universities for Research in Astronomy (AURA), and the U.S. National Science Foundation.

The Palomar MGSU was developed with support from the National Science Foundation (grant AST0096928, R. Dekany, PI), Caltech Optical Observatories, and the TMT Project.

\section{References}

1. B. L. Ellerbroek and F. J. Rigaut, "Scaling multiconjugate adaptive optics performance estimates to extremely large telescopes," in Adaptive Optical Systems Technology, P. L. Wizinowich, ed., Proc. SPIE, 4007, 1088-1099 (2000).

2. A. Tokovinin, "Seeing improvement with ground-layer adaptive optics," Publ. Astron. Soc. Pac. 116, 941-951 (2004).
3. R. W. Wilson, "SLODAR: measuring optical turbulence altitude with a Shack-Hartmann wavefront sensor," Mon. Not. R. Astron. Soc. 337, 103-108 (2002).

4. T. Butterley, R. W. Wilson, and M. Sarazin, "Determination of the profile of atmospheric optical turbulence strength from SLODAR data," Mon. Not. R. Astron. Soc. 369, 835-845 (2006).

5. R. W. Wilson, J. Bate, J. C. Guerra, N. N. Hubin, M. Sarazin, and C. D. Saunter, "Development of a portable SLODAR turbulence profiler," in Advancements in Adaptive Optics, D. B. Calia, B. L. Ellerbroek, and R. Ragazzoni, eds., Proc. SPIE 5490, 758-765 (2004).

6. L. Jolissaint, O. Keskin, C. Bradley, B. Wallace, and A. Hilton, "Multiple-layer optical turbulence generator principle and SLODAR characterization: preliminary results," in Optics in Atmospheric Propagation and Adaptive Systems VII, J. D. Gonglewski and K. Stein, eds., Proc. SPIE 5572, 256-261 (2004).

7. G. D. Love, C. N. Dunlop, S. Patrick, C. D. Saunter, R. W. Wilson, and C. Wright, "Horizontal turbulence measurements using SLODAR," in Atmospheric Optical Modeling, Measurement, and Simulation, S. M. Doss-Hammel and A. Kohnle, eds., Proc. SPIE 5891, 27-32 (2005).

8. V. Velur, R. C. Flicker, B. C. Platt, M. C. Britton, R. G. Dekany, M. Troy, J. E. Roberts, J. C. Shelton, and J. Hickey, "Multiple guide star tomography demonstration at Palomar observatory," in Advances in Adaptive Optics II, B. L. Ellerbroek and D. Bonaccini Calia, eds., Proc. SPIE 6272, 627258 (2006).

9. P. B. Stetson, "DAOPHOT-a computer program for crowdedfield stellar photometry," Publ. Astron. Soc. Pac. 99, 191-222 (1987).

10. W. H. Press, S. A. Teukolsky, W. T. Vetterling, and B. P. Flannery, Numerical Recipes in FORTRAN. The Art of Scientific Computing, 2nd ed. (Cambridge Press, 1992).

11. D. L. Fried, "Statistics of a geometric representation of wavefront distortion,” J. Opt. Soc. Am. 55, 1427-1435 (1965).

12. V. Kornilov, A. A. Tokovinin, O. Vozyakova, A. Zaitsev, N. Shatsky, S. F. Potanin, and M. S. Sarazin, "MASs: a monitor of the vertical turbulence distribution," in Adaptive Optical System Technologies II, P. L. Wizinowich and D. Bonaccini, eds., Proc. SPIE 4839, 837-845 (2003).

13. G. I. Taylor, "The spectrum of turbulence," Proc. R. Soc. London, Ser. A 164, 476-490 (1938).

14. M. Schöck and E. J. Spillar, "Method for a quantitative investigation of the frozen flow hypothesis," J. Opt. Soc. Am. A 17, 1650-1658 (2000). 